\title{
Identification des compartiments responsables de la qualité des eaux de surface d'un petit bassin versant du centre du Nouveau-Brunswick (Canada): application et analyse du modèle hydrochimique EMMA \\ End-members contributions to steamwater quality of a small catchment in central New Brunswick, Canada: verification of the applicability of the hydrochemical model EMMA
}

\author{
N. Bélanger, W. H. Hendershot, M. Bouchard et S. Jolicoeur
}

Volume 11, numéro 1, 1998

URI : https://id.erudit.org/iderudit/705300ar

DOI : https://doi.org/10.7202/705300ar

Aller au sommaire du numéro

Éditeur(s)

Université du Québec - INRS-Eau, Terre et Environnement (INRS-ETE)

ISSN

0992-7158 (imprimé)

1718-8598 (numérique)

Découvrir la revue

Citer cet article

Bélanger, N., Hendershot, W. H., Bouchard, M. \& Jolicoeur, S. (1998). Identification des compartiments responsables de la qualité des eaux de surface d'un petit bassin versant du centre du Nouveau-Brunswick (Canada): application et analyse du modèle hydrochimique EMMA. Revue des sciences de l'eau / Journal of Water Science, 11(1), 117-137. https://doi.org/10.7202/705300ar

\section{Résumé de l'article}

Cette étude a été réalisée dans le cadre d'un projet multidisciplinaire sur la gestion et la protection de l'habitat des salmonidés et sur l'évaluation des perturbations que subissent les habitats de ces poissons dans les eaux courantes suite aux coupes forestières et à la construction de routes. Afin d'identifier les voies d'écoulement responsables de la qualité des eaux de surface d'un petit bassin versant forestier, une étude approfondie a été entreprise sur l'évolution de la qualité de l'eau de pluie lors de son passage à travers la phytocénose et la couverture pédologique jusqu'au ruisseau. La signature chimique des compartiments du bassin versant servira d'intrant quant à l'application et l'analyse du modèle EMMA (end-members mixing analysis).

La signature chimique de l'eau du ruisseau s'explique par un graphe x-y (graphe de mélange) sur lequel la composition chimique des compartiments et celle du ruisseau sont reportées. Si trois compartiments circonscrivent la signature chimique du cours d'eau, alors on peut émettre l'hypothèse que ces compartiments se mélangent de façon conservatrice pour donner la qualité des eaux de surface du bassin versant. Plusieurs traceurs (conductivité électrique, $\mathrm{SO}_{4}{ }^{2-}, \mathrm{Cl}^{-}, \mathrm{NO}_{3}{ }_{3}^{-}, \mathrm{K}^{+}, \mathrm{Al}_{+}$et $\mathrm{Fe}_{+}$) naturels n'ont pas servi à l'identification des compartiments parce que le modèle ne tient pas compte de certaines conditions, tels l'activité biologique, l'état hydrique des profils, etc. Seuls le $\mathrm{pH}, \mathrm{Na}^{+}, \mathrm{Ca}^{2+}, \mathrm{Mg}^{2+}$ et $\mathrm{SiO}_{2}$ se sont avérés des traceurs utiles.

La nappe phréatique a été incluse par défaut dans le modèle puisqu'il était connu qu'elle assurait la base de l'écoulement du cours d'eau en tout temps de l'année. Les sols de la plaine d'inondation semblent également prendre part à la qualité de l'eau du ruisseau, particulièrement les horizons B podzoliques, lesquels sont saturés d'eau pendant toute la période sans gel. C'est donc dire que l'écoulement de l'eau souterraine et l'écoulement hypodermique au niveau des horizons B de la plaine d'inondation sont les voies d'écoulement qui expliquent le mieux la qualité des eaux de surface du bassin versant.

Toutefois, la séparation de l'hydrogramme par l'équation du bilan massique a montré qu'un modèle à trois réservoirs (nappe phréatique, horizons B des versants sud et nord) ne peut pas donner des résultats satisfaisants quant à la simulation de la charge chimique des eaux de surface. Le modèle élimine systématiquement trop de compartiments pouvant s'avérer explicatifs de la qualité de l'eau du ruisseau. Un modèle mécaniste développé à partir des variations du niveau de la nappe phréatique, de la conductivité hydraulique et de la composition chimique des solutions de sol permettrait de reproduire plus rigoureusement l'hydrogramme du ruisseau. Le modèle EMMA demeure tout de même un bon outil pour réfuter ou confirmer une hypothèse de recherche car il met clairement en relation la composition chimique des compartiments à celle du ruisseau et enlève parfois tout doute quant à l'action d'un processus susceptible d'alimenter le cours d'eau.
Ce document est protégé par la loi sur le droit d'auteur. L’utilisation des services d'Érudit (y compris la reproduction) est assujettie à sa politique d'utilisation que vous pouvez consulter en ligne.

https://apropos.erudit.org/fr/usagers/politique-dutilisation/ 


\title{
Identification des compartiments responsables de la qualité des eaux de surface d'un petit bassin versant du centre du Nouveau-Brunswick (Canada) : application et analyse du modèle hydrochimique EMMA
}

\author{
End-member contributions to streamwater quality of a small \\ catchment in central New Brunswick, Canada: verification \\ of the applicability of the hydrochemical model EMMA
}

\section{N. BÉLANGER ${ }^{1}$, W.H. HENDERSHOT ${ }^{1}$, M. BOUCHARD ${ }^{2}$ et S. JOLICOEUR ${ }^{3}$}

Reçu le 26 septembre 1996, accepté le 4 novembre 1997*.

\section{SUMMARY}

Intense forest harvesting is suspected as a cause of soil acidification. Inputs of acidity into the soil system may lead to high concentrations of metal ion species in the soil solution and surface waters. Some of these metal ions, e.g., $\mathrm{Al}^{3+}$, can cause toxic responses to fish and aquatic invertebrates. Timber-induced soil and surface water acidification is considered to be a short-lived phenomenon during the growing season following the cut. Vegetation loss could mean increased frequency of high $\mathrm{Al}^{3+}$ and $\mathrm{H}^{+}$concentrations in stream water rather than increased mean levels.

Many types of tracers are useful for hydrograph separation. Isotopic (e.g., deuterium and tritium) and natural chemical tracers (e.g., $\mathrm{pH}, \mathrm{Cl}^{-}, \mathrm{SiO}_{2}$ ) have been used extensively to interpret chemical data gathered in catchment studies. The ability of computer simulation models to reproduce the hydrograph and chemical species in streamwater varies. In some cases, the use of too many hydrological parameters (i.e., over-parameterization) can make the validation of reactions responsible for streamwater chemistry almost impossible. Recently, advances in hydrological modelling have been made by considering that streamwater chemistry is a mixture of groundwater and soil solutions at different depths. One model that originates from this hypothesis is EMMA (end-member mixing analysis). Chemical species that are variable with depth within a same soil profile were shown to be highly correlated with streamwater discharge. Generally, chemical species that show high concentrations in surface horizons increase in streamwater during high flow, whereas chemical species found in high concentrations in lower horizons are higher during low flow.

1. Department of Natural Resource Sciences, McGill University, Macdonald Campus, Sainte-Anne-de-Bellevue, QC, Canada, H9X 3 V9.

2. Département de Géographie, Université du Québec à Montréal, C.P.8888, Succ. Centre-Ville, Montréal, QC, Canada, $\mathrm{H} 3 \mathrm{C} 3 \mathrm{P} 8$.

3. Département d'Histoire et de Géographie, Université de Moncton, Moncton, NB, Canada, E1A 3E9.

* Les commentaires seront reçus jusqu'au 6 novembre 1998. 
In order to identify end-members that can potentially contribute to streamwater chemistry of a small catchment in central New Brunswick, we investigated the chemistry changes of rainwater entering the catchment, passing through vegetation and soils and reaching the stream channel. The chemical composition of the catchment's end-members will serve as input in order to run and analyse the EMMA model. Furthermore, a better knowledge of water flowpaths that dominate in the catchment could be valuable information for the Department of Fisheries and Oceans, who, in 1990, initiated a multidisciplinary project on 1) the protection and management of the salmonid habitat, and 2) the effects of forest harvesting and road construction on the freshwater habitats of these fish. Harvest operations are planned from 1996 until 1999.

Streamwater chemistry is explained by a $x-y$ graph (mixing diagram) on which the end-members and streamwater chemical composition are plotted. Because end-member chemistry is stable over time and space, mean values of tracers are plotted on the mixing diagram. Streamwater chemical compositions have all been plotted on the graph since they vary significantly with flow. If the chemical composition of three end-members enclose the streamwater chemical composition, then it can be assumed that these end-members mix conservatively to produce streamwater chemistry. If two chemical species mix non-conservatively, then the model will not accurately indicate the relative contribution of each end-member. Generally, the mixing diagram does not validate conservative mixing, but it can be used to test a mixing hypothesis. For example, if streamwater chemistry falls largely outside the end-members chemical composition, then at least one end-member is incorrectly characterized (or missing), or the end-members do not mix conservatively.

The relative contribution of selected end-members are obtain from the mass balance equation: $C_{t} Q_{t}=C_{1} Q_{1}+C_{2} Q_{2}+C_{3} Q_{3}$, where 1,2 and 3 refer to the three end-members, $\mathrm{C} 1,2$ and 3 are the soil water concentrations of conservative elements for each end-member, and Q1,2 and 3 are the amounts of soil water for a given end-member. With this equation, the concentrations of a number of elements for each end-member $\left(C_{1}, C_{2}, C_{3}\right)$ are used simultaneously to estimate a single value for each $Q_{1}, Q_{2}$ and $Q_{3}$. Since we want to quantify the contribution of each end-member to the total stream discharge, i.e., the mix of the three end-members, $Q_{t}$ is set to 1 . Once values for $Q_{1}, Q_{2}$ and $Q_{3}$ are calculated, the results are interpreted in terms of a hydrograph separation to show the contribution from each end-member to the overall stream discharge.

Two soil toposequences that correspond to typical soil profiles along the northern and southern hillslopes were selected. From June to November 1995, wet deposition, throughfall, soil solutions at four depths and streamwater were collected. Samples were analyzed for $\mathrm{pH}$, electrical conductivity, $\mathrm{Na}^{+}, \mathrm{Ca}^{2+}, \mathrm{Mg}^{2+}$, $\mathrm{K}^{+}, \mathrm{SO}_{4}{ }^{2-}, \mathrm{Cr}, \mathrm{NO}_{3}^{-}, \mathrm{SiO}_{2}, \mathrm{Al}_{\mathrm{t}}$ and $\mathrm{Fe}_{\mathrm{t}}$.

Many natural tracers (electrical conductivity, $\mathrm{SO}_{4}{ }^{2-}, \mathrm{Cl}^{-}, \mathrm{NO}_{3}^{-}, \mathrm{K}^{+}, \mathrm{AI}_{4}$ and $\mathrm{Fe}_{t}$ ) have not been used to identify end-members because the model does not always consider adequately some conditions or processes that go on in the catchment, e.g., biological activity and $E h$. Because they vary considerably with depth, solution $\mathrm{pH}, \mathrm{Na}^{+}, \mathrm{Ca}^{2+}, \mathrm{Mg}^{2+}$ and $\mathrm{SiO}_{2}$ have been shown to be useful tracers.

Groundwater has been included in every diagram as one of the three endmembers mixing conservatively to produce streamwater since it is certain that it contributes a large portion to the total discharge under any hydrological condition. Soils along the stream seem to contribute the rest of the streamwater chemistry, particularly B horizons which are submerged all summer by groundwater. Thus, groundwater and subsurface flow at the base of the soil profiles along the stream seem to be the principal flow mechanisms that control streamwater chemistry in the catchment. 
However, hydrograph separation shows that a three end-member model (i.e., groundwater, B horizons from the northern and southern hillslopes) is not enough to simulate streamwater chemistry. Saturated subsurface flow in the B horizons from both sides of the stream should contribute approximately the same amount to the total discharge since groundwater affects both end-members throughout the growing season. In that respect, groundwater level fluctuations at this depth of the soil profiles should not be considered as a cause of this discrepancy. What can be said at this point is that one end-member that is incorrectly defined in space, and that has a similar chemical composition to saturated subsurface flow coming from the southern hillslope, is the primary source (with groundwater) of stream discharge during events. It is thus better to interpret this information in terms of solution type rather than in terms of physical origin (northern or southern hillslope). In this manner, the stream water is provided by both hillslopes.

In conclusion, the model eliminates systematically too many end-members that could partially explain streamwater chemistry. Results show that a more complex mixture is necessary to reproduce streamwater chemistry. A mechanistic model based on groundwater level fuctuations, hydraulic conductivity and soil solution chemistry would possibly have better success in reproducing the stream hydrograph. However, EMMA remains a useful tool to refute or confirm the possible action of a flow mechanism by correlating the chemical composition of end-members with streamwater chemistry.

Key words: EMMA, end-member, hydrochemical modelling, mixing diagram.

Cette étude a été réalisée dans le cadre d'un projet multidisciplinaire sur la gestion et la protection de l'habitat des salmonidés et sur l'évaluation des perturbations que subissent les habitats de ces poissons dans les eaux courantes suite aux coupes forestières et à la construction de routes. Afin d'identifier les voies d'écoulement responsables de la qualité des eaux de surface d'un petit bassin versant forestier, une étude approfondie a été entreprise sur l'évolution de la qualité de l'eau de pluie lors de son passage à travers la phytocénose et la couverture pédologique jusqu'au ruisseau. La signature chimique des compartiments du bassin versant servira d'intrant quant à l'application et l'analyse du modèle EMMA (end-members mixing analysis).

La signature chimique de l'eau du ruisseau s'explique par un graphe x-y (graphe de mélange) sur lequel la composition chimique des compartiments et celle du ruisseau sont reportées. Si trois compartiments circonscrivent la signature chimique du cours d'eau, alors on peut émettre l'hypothèse que ces compartiments se mélangent de façon conservatrice pour donner la qualité des eaux de surface du bassin versant. Plusieurs traceurs (conductivité électrique, $\mathrm{SO}_{4}{ }^{2-}$, $\mathrm{Cl}^{-}, \mathrm{NO}_{3}^{-}, \mathrm{K}^{+}, \mathrm{Al}_{\mathrm{t}}$ et $\mathrm{Fe}_{\mathrm{t}}$ ) naturels n'ont pas servi à l'identification des compartiments parce que le modèle ne tient pas compte de certaines conditions, tels l'activité biologique, l'état hydrique des profils, etc. Seuls le $\mathbf{p H}, \mathrm{Na}^{+}, \mathrm{Ca}^{2+}$, $\mathrm{Mg}^{2+}, \mathrm{SiO}_{2}$ se sont avérés des traceurs utiles.

La nappe phréatique a été incluse par défaut dans le modèle puisqu'il était connu qu'elle assurait la base de l'écoulement du cours d'eau en tout temps de l'année. Les sols de la plaine d'inondation semblent également prendre part à la qualité de l'eau du ruisseau, particulièrement les horizons B podzoliques, lesquels sont saturés d'eau pendant toute la période sans gel. C'est donc dire que l'écoulement de l'eau souterraine et l'écoulement hypodermique au niveau des horizons B de la plaine d'inondation sont les voies d'écoulement qui expliquent le mieux la qualité des eaux de surface du bassin versant. 


\begin{abstract}
Toutefois, la séparation de l'hydrogramme par l'équation du bilan massique a montré qu'un modèle à trois réservoirs (nappe phréatique, horizons B des versants sud et nord) ne peut pas donner des résultats satisfaisants quant à la simulation de la charge chimique des eaux de surface. Le modèle élimine systématiquement trop de compartiments pouvant s'avérer explicatifs de la qualité de l'eau du ruisseau. Un modèle mécaniste développé à partir des variations du niveau de la nappe phréatique, de la conductivité hydraulique et de la composition chimique des solutions de sol permettrait de reproduire plus rigoureusement l'hydrogramme du ruisseau. Le modèle EMMA demeure tout de même un bon outil pour réfuter ou confirmer une hypothèse de recherche car il met clairement en relation la composition chimique des compartiments à celle du ruisseau et enlève parfois tout doute quant à l'action d'un processus susceptible d'alimenter le cours d'eau.
\end{abstract}

Mots-clés : compartiment, EMMA, graphe de mélange, modélisation hydrochimique.

\title{
1 - INTRODUCTION
}

\subsection{Aperçu du problème}

Au Canada, le secteur de l'industrie forestière fait l'objet de nombreuses polémiques. Les impacts des coupes forestières sont nombreux : érosion hydrique, perte de productivité des sols, problèmes de régénération de la forêt, etc. Encore tout récemment, NODVIN et al. (1988) et PLAMONDON (1993) ont montré que l'exploitation forestière expliquait localement la chute du pH des solutions de sol et des eaux de surface suite à une intensification du processus de nitrification. La chute du $\mathrm{pH}$ des solutions de sol favoriserait la mobilisation d'ions métalliques jusqu'au cours d'eau, particulièrement $\mathrm{Al}^{3+}$, lesquels sont toxiques pour certaines espèces de poissons et invertébrés aquatiques (BAKER et SCHOFIELD, 1982 ; HALL et al., 1985 ; SCHINDLER, 1988). D'après LIKENS et al. (1970), l'acidification des sols et des eaux de surface qui suit la perte du couvert forestier serait un phénomène localisé dans le temps, soit quelques semaines après le début de la première saison estivale suivant la coupe des arbres. L'effet le plus important de la perte de la phytocénose sur la qualité de l'eau de l'exutoire serait celui d'une augmentation des fréquences de fortes concentrations en $\mathrm{Al}^{3+}$ et $\mathrm{H}^{+}$, plutôt que d'une hausse des teneurs moyennes.

\subsection{Développement théorique}

Plusieurs équipes de recherche ont tenté d'expliquer la composition chimique des eaux de surface par celle des solutions de sol. Les traceurs isotopiques stables (deuterium, tritium et $O_{18}$ ) ont été utilisés par plusieurs auteurs (DINCER et al., 1970 ; MARTINEC, 1975 ; PEARCE et al., 1986 ; MAULÉ et STEIN, 1990) afin de calculer la contribution relative de l'eau stockée dans le bassin versant entre les averses (vieille eau) et de l'eau de pluie ou de fonte (jeune eau) au débit total du cours d'eau. La plupart de ces études ont indiqué que la "vieille eau " domine le cours d'eau lors de fortes décharges.

Bien que la distinction entre la « vieille eau » et la " jeune eau » soit d'un intérêt académique, elle ne permet pas d'identifier avec précision les compartiments contribuant à la qualité des eaux de surface. C'est pourquoi HENDERSHOT et al. 
(1992) ont suggéré d'utiliser des traceurs qui identifient clairement les réactions chimiques aux différentes profondeurs des sols. Cette approche permettrait de développer un modèle explicite mettant en relation l'acquisition de la charge chimique des eaux de surface et la couverture pédologique. PINDER et JONES (1969) ont proposé d'utiliser des traceurs chimiques naturels, tels la conductivité électrique, le $\mathrm{pH}, \mathrm{Cl}^{-}, \mathrm{SO}_{4}{ }^{2-}, \mathrm{Ca}^{2+}, \mathrm{Mg}^{2+}$ et $\mathrm{Na}^{+}$. Toutefois, l'interprétation des résultats de la séparation de l'hydrogramme s'est avérée compliquée puisque les traceurs naturels ont interagi avec le soubassement rocheux et les formations superficielles du bassin versant. Pourtant, en utilisant $\mathrm{SiO}_{2}$ comme traceur, HOOPER et SHOEMAKER (1986) ont fait la corrélation avec $\mathrm{O}_{18}$ et conclurent que $\mathrm{SiO}_{2}$ était un élément conservateur (i.e., aucune interaction avec le sol, les solutions de sol ou le ruisseau) et, par conséquent, convenable pour la séparation de l'hydrogramme.

Tout récemment, l'utilisation combinée des modèles hydrologiques et des modèles chimiques, i.e., les modèles hydrochimiques, a permis de reproduire assez rigoureusement l'hydrogramme et les chémogrammes de plusieurs espèces chimiques. Dans l'ensemble, les modèles hydrochimiques, tels ILWAS (GHEFINI et al., 1985), BIRKENES (CHRISTOPHERSEN et al., 1982) et PULSE (BERGSTRÖM et al., 1985), sont des représentations conformes aux sols des bassins versants à l'étude, mais quelques paramètres sont ajustés de façon à ce que les résultats de la simulation correspondent aux données sur le terrain (HENDERSHOT et al., 1992). Cependant, les modèles ne contiennent pas toutes les réactions chimiques pouvant avoir lieu dans les sols, mais ils incorporent les plus importantes, dont l'adsorption des sulfates et l'échange cationique. De plus, HOOPER et al. (1988) ont montré que l'incorporation d'un surnombre de paramètres hydrologiques aux modèles hydrochimiques (i.e., surparamétrisation), dont il n'est pas toujours possible de ne déterminer qu'une seule valeur à partir des données de terrain, complique la validation des réactions chimiques qui pourraient s'avérer explicatives de la composition chimique des eaux de surface.

Encore aujourd'hui, la formalisation des processus hydrologiques et chimiques diffère trop d'un modèle à l'autre pour qu'il soit possible de synthétiser l'information et tirer des conclusions quant à l'origine de la composition chimique des eaux de surface des petits bassins versants. Lapproche de HOOPER et al. (1990) et CHRISTOPHERSEN et al. (1990) est différente de celle décrite précédemment parce qu'elle donne aux modèles hydrochimiques une structure hydrologique moins imposante. La technique se base sur l'hypothèse voulant que la qualité des eaux de surface est le résultat du mélange de la nappe phréatique et des solutions de sol à différentes profondeurs, d'où son nom de end-members mixing analysis (EMMA). Par exemple, NEAL et al. (1986) ont observé que les espèces chimiques les mieux corrélées au débit du cours d'eau sont celles dont les concentrations sont les plus variables à travers le profil pédologique. Ainsi, les espèces chimiques dont les concentrations sont élevées dans les percolats des horizons de surface (e.g., $\mathrm{H}^{+}$et $\mathrm{Al}_{\mathrm{i}}$ ) voient leur teneur augmenter dans le cours d'eau en période de crue, alors que les teneurs des espèces chimiques qui se retrouvent en forte concentration dans les solutions des horizons inférieurs (e.g., $\mathrm{Ca}^{2+}$, $\mathrm{SiO}_{2}$ et $\mathrm{HCO}_{3}{ }^{-}$) sont plus élevées en période d'étiage.

CHRISTOPHERSEN et al. (1990) ont appliqué le modèle EMMA à des données provenant des bassins versants de Birkenes (Norvège) et de Plynlimon (pays de Galles). Dans les deux cas, il semble que les compartiments qui ont été chimiquement caractérisés n'aient pas été suffisants pour reproduire la signature chimique des eaux de surface. Au contraire, HOOPER et al. (1990) ont réussi à expliquer la 
qualité des eaux de surface du bassin versant de Panola Mountain (Georgie, Etats-Unis) par le mélange des eaux de la nappe phréatique, des horizons organiques à proximité du cours d'eau et d'un horizon minéral de mi-versant.

\subsection{Objectifs}

C'est dans le but d'identifier les voies d'écoulement responsables de la qualité des eaux de surface d'un petit bassin versant forestier qu'une étude approfondie a été entreprise sur l'évolution de la composition chimique de l'eau de pluie lors de son passage à travers la phytocénose et la couverture pédologique jusqu'au ruisseau. La signature chimique des compartiments du bassin versant servira d'intrant quant à l'application et l'analyse du modèle EMMA. La détermination des voies d'écoulement servira d'information précieuse au Ministère des Pêches et des Océans, lequel y a entamé, en 1990, un projet multidisciplinaire sur la gestion et la protection de l'habitat des salmonidés et sur l'évaluation des perturbations que subissent les habitats de ces poissons dans les eaux courantes suite aux coupes forestières et à la construction de routes prévues entre 1996 et 1999 (CUNJAK et al., 1990).

\section{2 - PRÉSENTATION DU BASSIN VERSANT}

Le bassin versant du ruisseau Catamaran est situé dans le centre du NouveauBrunswick $\left(46^{\circ} 52^{\prime} 7^{\prime \prime} \mathrm{N}, 66^{\circ} 06^{\circ} 0^{\circ} \mathrm{O}\right), 100 \mathrm{~km}$ à l'ouest du golfe du Saint-Laurent (fig. 1). Seule la partie amont du bassin versant de l'affluent $n^{\circ} 1$ du ruisseau Catamaran a été retenue pour l'étude. D'une superficie d'environ $3,75 \mathrm{~km}^{2}$, celle-ci est orientée du SO au NE et se résume à un versant nord relativement court et à un versant sud plus ou moins régulier (fig. 2). Dans sa section centrale, des ruptures de pente de 6 à 8 mètres de dénivelée limitent la plaine d'inondation, de part et d'autre. La dénivelée maximale dans le sous-bassin est de plus de $130 \mathrm{~m}$, mais elle se fait sur une distance d'environ $4,5 \mathrm{~km}$. Les affleurements rocheux se limitent principalement aux ruptures de pente. Is sont constitués de roches volcano-sédimentaires, notamment des basaltes en coussins ainsi que des slates et des cherts ferromanganifères et graphitiques. Le bassin versant est recouvert d'une moraine de texture sableuse variant de 0,5 à $3 \mathrm{~m}$ d'épaisseur, constituée par un till de fond compact parfois surmonté d'un till d'ablation moins compact (RAMPTON et al., 1984). Ces sédiments, provenant du centre de dispersion glaciaire du nord du Maine, à l'ouest, contiennent des matériaux exotiques, par exemple des cailloux de granite. Dans la plaine d'inondation, il y a des sédiments glaciaires et des alluvions actuelles. Ils sont de texture limoneuses à sablo-graveleuse.

Le climat de la région est de type tempéré froid et humide, avec une température moyenne annuelle de $4,3^{\circ} \mathrm{C}$. Le mois le plus chaud est juillet $\left(18,8^{\circ} \mathrm{C}\right)$ et le mois le plus froid est janvier $\left(-10,7^{\circ} \mathrm{C}\right)$. Les précipitations moyennes annuelles sont d'environ $1000 \mathrm{~mm}$, dont $72 \%$ tombent sous forme de pluie (Transports Canada, 1984).

Le bassin est couvert à $65 \%$ de conifères et à $35 \%$ de feuillus. Toutefois, cette proportion n'est pas homogène pour l'ensemble du bassin versant. Au sommet du versant sud, les feuillus sont plus nombreux que les résineux, alors que dans la plupart des autres secteurs ce sont les résineux, tels le cèdre de l'est 


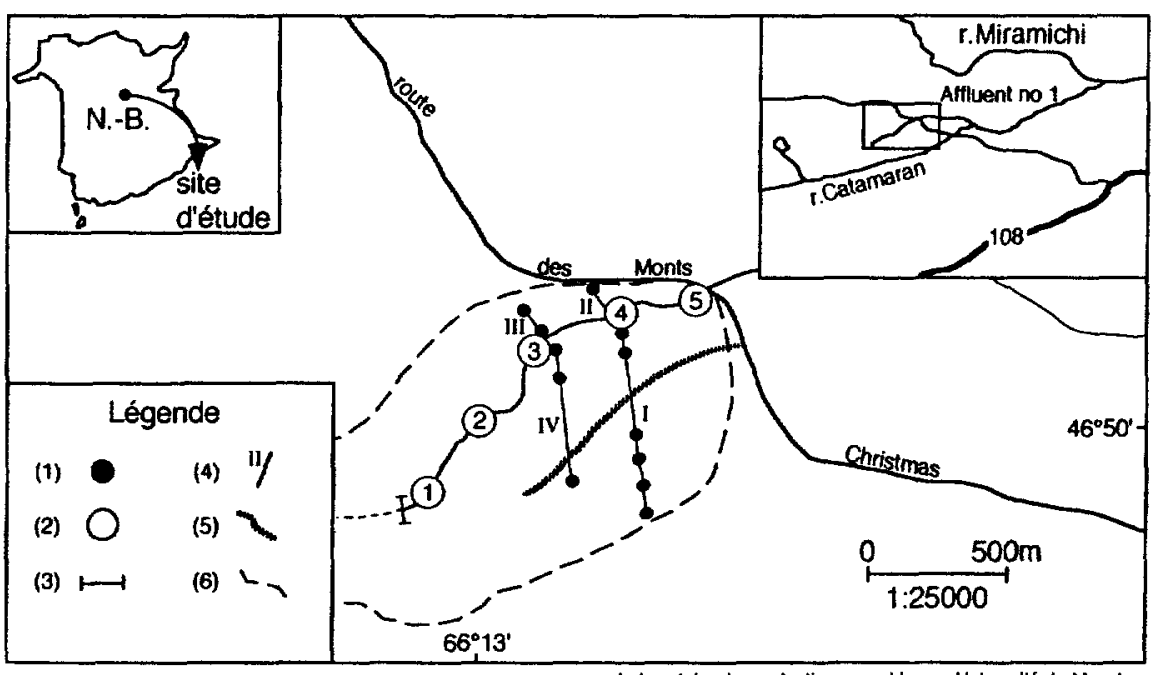

Laboratoire de productions graphiques, Universtté de Moncton

(1) Pédon, (2) Site d'échantillonnage du cours d'eau, (3) Barrage de castor, (4) Toposéquence, (5) Ancien chemin forestier, (6) Limite du bassin versant.

(1) Soil profile, (2) Streamwater sampling site, (3) Beaver dam, (4) Toposequence, (5) Ord forest road, (6) Catchment limit.

Figure 1 Localisation du bassin versant de l'affluent $n^{\circ} 1$ du ruisseau Catamaran et des sites d'étude.

Location of Catamaran brook tributary $n^{\circ} 1$ catchment and study sites.

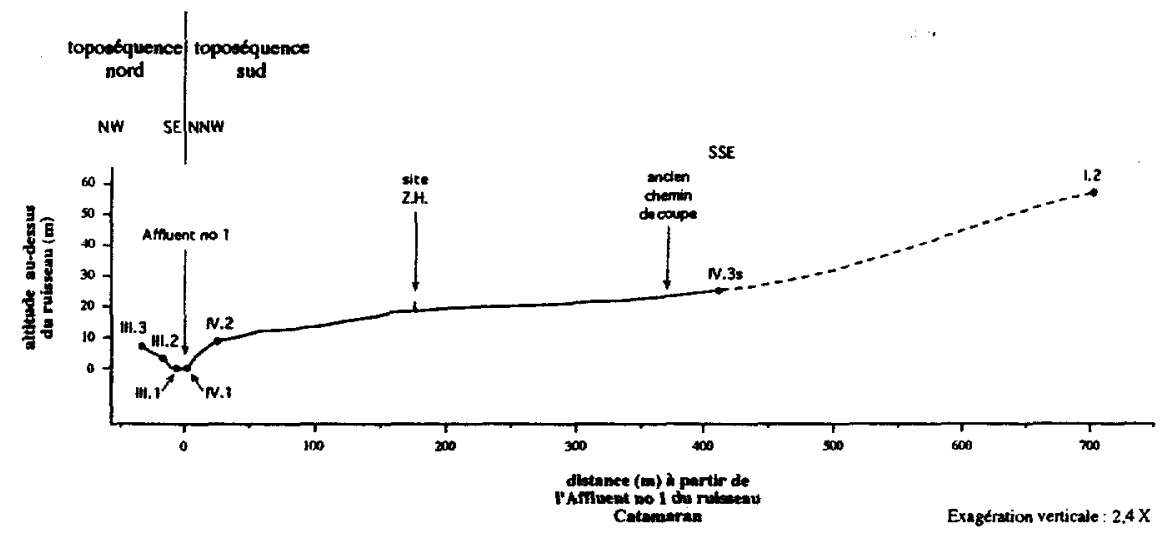

Figure 2 Coupe schématisée des toposéquences nord et sud au travers du bassin versant de l'affluent $n^{\circ} 1$ du ruisseau Catamaran.

Cross-section of the north and south toposequences throughout Catamaran brook tributary $n^{\circ} 1$ catchment.

(Thuja occidentalis L.), le sapin baumier (Abies balsamea (L.) Mill.) et les épinettes rouge (Picea rubens Sarg.) et noire (Picea mariana (Mill.) BSP.), qui constituent l'essentiel de la forêt. L'âge moyen des peuplements est de soixante ans, 
mais il y a des secteurs où des traces de coupe forestière sont visibles et où la forêt est nettement plus jeune et moins dense. En de nombreux endroits, les vieilles souches de grands cèdres de l'est sont fréquentes et témoignent d'une perturbation du couvert forestier par l'exploitation forestière.

En règle générale, le bassin versant de l'affluent $n^{\circ} 1$ est recouvert de podzols, saut dans les endroits mal drainés où se sont développés des gleysols. Toutefois, les traits pédologiques du bassin, surtout le long du versant sud, montrent très bien qu'il faut l'étudier à différentes échelles. JOLICOEUR et al. (1996) ont divisé le versant sud en quatre sections (fig. 2):

i) un segment en pente modérée débutant à la limite du bassin versant. Le sommet du versant y est recouvert d'une forêt dominée par le hêtre (Fagus grandifolia Ehrh.), mais les sapins, les épinettes et de très vieux cèdres sont aussi présents. Les sols de ce géotope sont des podzols humo-ferriques orthiques (pédon 1.2, tabl. 1). Les caractères sont proches de ceux des brunisols dystriques, avec notamment un horizon Ahe bien net, mais les caractéristiques morphologiques et chimiques des horizons $\mathrm{Bf}$ satisfont aux exigences des horizons podzoliques ;

ii) une vaste zone relativement plane où les conifères sont matures et dominants. À la hauteur de la toposéquence IV, ce segment constitue plus de la moitié du versant sud. Des conditions d'hydromorphie, même en juillet, ont été observées à l'été 1994. Dans de telles conditions, la matière organique s'accumule et des gleysols se développent. Le micro-relief en creux et en bosses de l'ordre du décimètre permet le développement de gleysols régosoliques dans les creux mal drainés, alors que des podzols humo-ferriques orthiques occupent les bosses, mieux drainées (pédon IV.3s, tabl. 1) ;

iii) un segment où la pente redevient perceptible et où le drainage est meilleur. Les conifères sont dominants. On y retrouve des podzols ferro-humiques orthiques, comme le pédon IV.2 (tab). 1). Ces sols présentent des horizons éluviaux (Ae) faisant parfois plus de dix centimètres d'épaisseur, ainsi que des horizons Bhf très nets;

iv) une plaine d'inondation située à quelques décimètres au-dessus du niveau du ruisseau drainant le bassin versant. La plaine est entrelacée de chenaux plus ou moins occupés par le ruisseau selon les conditions hydrologiques. Elle est occupée par des podzols humo-ferriques gleyifiés (pédon IV.1, tabI. 1) et des gleysols orthiques (pédon III.1, tabl. 1). La texture joue ici un rôle: dans le till limono-argileux du pédon III.1, l'eau circule difficilement, favorisant la réduction et la gleyification, alors que les alluvions sablo-graveleuses du pédon IV.1, bien que la plupart du temps gorgées d'eau, restent mieux oxygénées.

II semble donc que ce soit le drainage qui détermine la distribution des types de sols sur le versant sud. Le micro-relief vient compliquer davantage cette distribution à une échelle plus fine. Le versant nord, de 250 mètres au plus, est relativement plus simple: on y retrouve des podzols humo-ferriques orthiques. Le pédon III.3 (tabl. 1) est en tous points comparable aux pédons I.2 et IV.3s.

\section{3 - MÉTHODOLOGIE ET TECHNIQUES D'ANALYSE}

La qualité de l'eau du ruisseau s'explique à partir d'un graphe $x-y$ dans lequel il faut incorporer les caractéristiques chimiques des compartiments et celles de 
Tableau 1 Propriétés physiques et chimiques des pédons du bassin versant de l'affluent $n^{\circ} 1$ du ruisseau Catamaran (Tiré de JOLicoeur et al., 1996).

Table 1 Physical and chemical properties of the soil profiles of Catamaran brook tributary $n^{\circ} 1$ catchment (from JOLICOEUR et al., 1996).

\begin{tabular}{|c|c|c|c|c|c|c|c|c|c|}
\hline \multirow{2}{*}{$\begin{array}{l}\text { Pédon et } \\
\text { type de sol }\end{array}$} & \multirow{2}{*}{ Horizon } & \multirow{2}{*}{$\begin{array}{l}\text { Profondeur } \\
\text { (cm) }\end{array}$} & \multirow{2}{*}{$\begin{array}{c}\text { Sables } \\
(\%)\end{array}$} & \multirow{2}{*}{$\begin{array}{c}\text { Limons } \\
(\%)\end{array}$} & \multirow{2}{*}{$\begin{array}{c}\text { Argiles } \\
(\%)\end{array}$} & \multirow{2}{*}{$\begin{array}{c}\text { Carbone } \\
\text { org. } \\
(\%)\end{array}$} & \multirow{2}{*}{$\underset{\left(\mathrm{H}_{2} \mathrm{O}\right)}{\mathrm{pH}}$} & \multicolumn{2}{|c|}{ Pyrophosphate } \\
\hline & & & & & & & & $\%$ Al & $\% \mathrm{Fe}$ \\
\hline \multirow{5}{*}{$\begin{array}{l}\text { Pédan } 1.2 \\
\text { (PHF.0) }\end{array}$} & LFH & $00-10$ & - & - & - & - & 4,5 & - & - \\
\hline & Anej & $10-13$ & 46,2 & 43,0 & 10,8 & 6,4 & 4,5 & 0,9 & 0,2 \\
\hline & $B f 1$ & $13-34$ & 50,2 & 43,2 & 6,7 & 2,3 & 5,3 & 1,1 & 1,3 \\
\hline & $B \nmid 2$ & $34-54$ & - & - & - & - & - & - & - \\
\hline & $c$ & $54-78$ & 59,4 & 35,9 & 4,7 & 0,9 & 5,6 & 0,1 & 0,2 \\
\hline \multirow{6}{*}{$\begin{array}{l}\text { Pédon IV.3s } \\
\text { (PHF.0) }\end{array}$} & LFH & $00-09$ & - & - & - & - & 4,9 & - & - \\
\hline & Ahej & $09-18$ & 41,1 & 43,0 & 15,9 & 14,6 & 4,9 & 1,5 & 2,2 \\
\hline & $B+1$ & $18-38$ & 63,3 & 33,2 & 3,5 & 2,5 & 4,9 & 0,9 & 1,9 \\
\hline & $B+2$ & $38-48$ & 67,7 & 30,3 & 2,0 & 3,2 & 5,1 & 1,0 & 2,0 \\
\hline & $B C$ & $48-58$ & 63,5 & 32,5 & 4,0 & 1,2 & 5,1 & 0,2 & 0,3 \\
\hline & $\mathrm{Cg}$ & $58-88$ & 54,8 & 36,0 & 9,1 & - & - & - & - \\
\hline \multirow{5}{*}{$\begin{array}{l}\text { Pédon IV.2 } \\
\text { (PHF.0) }\end{array}$} & LFH & $00-11$ & - & - & - & - & 4,0 & - & - \\
\hline & $\mathrm{Ae}$ & $11-22$ & 61,8 & 35,5 & 2,7 & 0,6 & 3,9 & 0,0 & 0,0 \\
\hline & Bhf & $22-32$ & 65,3 & 29,2 & 5,6 & 8,9 & 4,3 & 1,7 & 2,4 \\
\hline & $B f j$ & $32-41$ & 80,4 & 18,6 & 1,1 & 2,3 & 4,8 & 0,1 & 0,5 \\
\hline & C & $41-71$ & 65,5 & 31,4 & 3,1 & 0,7 & 4,9 & 0,1 & 0,2 \\
\hline \multirow{4}{*}{$\begin{array}{l}\text { Pédon IV.1 } \\
\text { (PHF.G) }\end{array}$} & LFH & $00-07$ & - & - & - & - & 5,0 & - & - \\
\hline & Ahej & $07-14$ & 55,6 & 42,2 & 2,2 & 10,5 & 5,2 & 0,9 & 0,6 \\
\hline & Btgj & $14-32$ & 71,3 & 25,8 & 2,9 & 1,8 & 5,3 & 0,2 & 0,2 \\
\hline & Coj & $32-43$ & 88,9 & 10,4 & 0,7 & 1,2 & 5,2 & 0,1 & 0,2 \\
\hline \multirow{6}{*}{$\begin{array}{l}\text { Pédon III.1 } \\
\text { (G.0) }\end{array}$} & LFH & $00-09$ & - & - & - & - & 4,7 & - & - \\
\hline & Ahej & $09-17$ & 59,8 & 31,8 & 8,5 & 6,3 & 5,2 & 1,0 & 0,6 \\
\hline & $\mathrm{Bg}$ & $17-43$ & 67,1 & 25,3 & 7,6 & 1,9 & 5,4 & 1,1 & 1,1 \\
\hline & $\mathrm{Bg}$ & 35 & - & - & - & - & 5,3 & - & - \\
\hline & (mar.) & $43-73$ & 59,5 & 29,8 & 10,7 & 0.9 & 5,5 & 0,8 & 0,6 \\
\hline & $\mathrm{Cg}$ & & & & & & & & \\
\hline \multirow{5}{*}{$\begin{array}{l}\text { Pédon Ill.3 } \\
\text { (PHF.0) }\end{array}$} & LFH & $00-07$ & - & - & - & - & 4,0 & - & - \\
\hline & $\mathrm{Ae}$ & $07-21$ & 69,1 & 26,4 & 4,5 . & 10,5 & 3,6 & 0,0 & 0,0 \\
\hline & $\mathrm{Bff}$ & $21-30$ & - & - & - & 2,6 & 4,6 & 0,4 & 2,1 \\
\hline & $B \nmid 2$ & $30-42$ & 71,0 & 28,0 & 1,0 & 2,2 & 4,9 & 0,2 & 0,6 \\
\hline & $C$ & $42-59$ & 67,4 & 30,7 & 2,0 & 0,4 & 5,2 & 0,0 & 0,1 \\
\hline
\end{tabular}

PHF.0 = podzol humo-ferrique orthique; PFH.0 = podzol ferro-humique orthique; PHF.G = podzol humo-ferrique gleyifié et $\mathrm{G} .0=$ gleysol orthique.

PHF. $O=$ Orthic humo-ferric podzol; $P F H . O=$ Orthic ferro-humic podzol; $P H F, G=$ Gleyified humo-ferric podzol and $G . O=$ Orthic gleysol.

l'eau du ruisseau (fig. 3). Chaque donnée sur le graphe représente un couple formé de traceurs chimiques naturels. Avec EMMA, toutes les possibilités d'association des traceurs sont considérées. Puisque la qualité de l'eau du ruisseau varie considérablement avec le débit, toutes les données sont reportées sur le graphe. Quant aux compartiments, seule la moyenne des traceurs y est reportée 


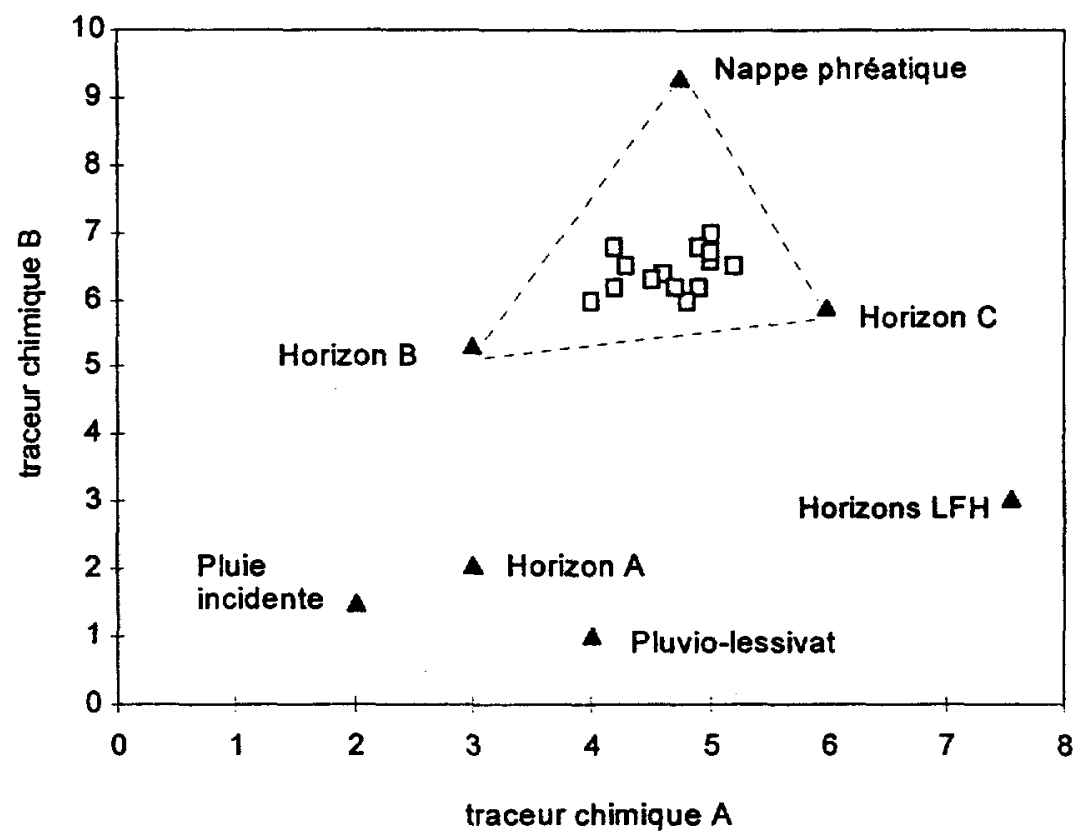

N.B. : Les échantillons du cours d'eau sont identifiés par des carrés et les compartiments par des triangles. N.B. : The streamwater chemistry is represented by squares and end-members by triangles.

Figure 3 Exemple d'un graphe de mélange.

Example of a mixing diagram.

parce qu'elle suffit pour représenter la variation de leurs caractéristiques chimiques dans le temps et lespace (CHRISTOPHERSEN et al., 1990). Pour que le mélange des compartiments soit conservateur, la signature chimique du cours d'eau doit s'étendre en dedans des compartiments retenus. Au total, trois compartiments sont retenus pour la simulation. À n'importe quel point dans le temps, la contribution des solutions de sol (i.e., compartiments) à l'écoulement du cours d'eau se mesure en utilisant deux espèces chimiques et la somme de la contribution des trois compartiments retenus égale à un (ou 100\%). Parce que les compartiments sont représentés par la moyenne des concentrations des solutions de sol, la contribution relative de chacun est retenu seulement si 1) la teneur d'un traceur diffère suffisamment entre les compartiments, et 2) la teneur des traceurs est suffisamment constante dans le temps et l'espace (CHRISTOPHERSEN et al., 1990). Si le mélange de deux espèces chimiques est non conservateur, alors le graphe n'indiquera pas avec exactitude la contribution relative de chaque compartiment au débit total du cours d'eau. CHRISTOPHERSEN et al. (1990) reconnaissent que le graphe ne valide pas le mélange conservateur, mais il peut au moins être utilisé pour réfuter l'hypothèse de mélange. Par exemple, si la signature chimique du cours d'eau s'étend significativement en dehors de celle des trois compartiments, au moins un compartiment est incorrectement caractérisé (ou manquant) et/ou le mélange des compartiments est non conservateur.

On détermine la contribution relative des compartiments à partir de l'équation du bilan massique suivante: $C_{t} Q_{t}=C_{1} Q_{1}+C_{2} Q_{2}+C_{3} Q_{3}$. 
où 1,2 et 3 se réfèrent aux trois compartiments identifiés à l'aide du graphe de mélange, $C(1,2$ et 3$)$ à la concentration moyenne des éléments conservateurs de chaque compartiment, et $\mathrm{Q}(1,2$ et 3$)$ à la contribution relative de chaque compartiment à l'écoulement total du ruisseau $\left(Q_{t}\right)$. À partir de cette équation, les concentrations des traceurs naturels conservateurs sont utilisées simultanément sous forme de matrice pour estimer une valeur unique pour $Q_{1}, Q_{2}$ et $Q_{3}$. Toutefois, certaines contraintes doivent être observées au cours de la procédure. Puisqu'il faut évaluer la contribution relative de chacun des compartiments à l'ècoulement total du cours d'eau, i.e., le mélange de ces réservoirs, alors $Q_{t}$ est égal à un (ou $100 \%$ ). La somme de $Q_{1}, Q_{2}$ et $Q_{3}$ doit égaler un; $Q_{1}, Q_{2}$ et $Q_{3}$ peuvent égaler zéro (aucune contribution), mais ne peuvent pas être négatifs. Si les valeurs obtenues pour $Q_{1}, Q_{2}$ et $Q_{3}$ sont négatives, le système déterminé est résolu à nouveau avec le $\mathrm{Q}$ négatif fixé à zéro.

Une fois que les valeurs de $Q_{1}, Q_{2}$ et $Q_{3}$ sont obtenues, les résultats sont interprétés sur un hydrogramme. L'hydrogramme montre alors la contribution relative de chaque compartiment en fonction des fluctuations du débit total du cours d'eau.

En octobre 1994, deux transects correspondant à des toposéquences de sols représentatives des versants sud et nord du bassin de l'affluent $n^{\circ} 1$ ont été sélectionnés. La toposéquence du versant sud est composée des pédons I.2, IV.3s, IV.2 et IV.1. Le pédon I.2 y a été inclus étant donné que les conditions de station étaient identiques sur l'ensemble du sommet du versant sud. La toposéquence du versant nord, beaucoup plus courte, est composée des pédons III.1 et III.3 (fig. 1 et 2 ).

De juin à novembre 1995, le jour suivant chaque pluie, il y a eu prélèvement des précipitations incidentes, des pluvio-lessivats, des solutions de sol et de l'eau du ruisseau. L'eau de pluie était recueillie à l'aide de pluvio-collecteurs composés d'un entonnoir en polyéthylène de $25 \mathrm{~cm}$ de diamètre communiquant à une bouteille en verre de $750 \mathrm{ml}$. Ceux-ci étaient maintenus constamment ouverts, mais étaient recouverts d'une mousseline pour éviter toute contamination par macération d'insectes, de débris végétaux, etc. L'échantillonnage des solutions de sol s'est fait à 4 profondeurs dans les six profils, soit sous les horizons organiques (LFH-Ah) et dans les horizons A, B et C. Les solutions des horizons organiques étaient recueillies au moyen de lysimètres passifs formés d'un entonnoir en polyéthylène de $25 \mathrm{~cm}$ de diamètre, recouvert d'une mousseline et d'un support quadrillé en polyéthylène et relié à une bouteille en verre de $750 \mathrm{ml}$. Les solutions des horizons A, B et C étaient prélevés au moyen de tubes en PVC de $4,5 \mathrm{~cm}$ de diamètre munis d'une bougie poreuse en porcelaine (Soil Water Sampler \# 1900 , Soil Moisture Equipment Co., Santa Barbara, Californie). C'est en exerçant une succion de 70 centibars que ces lysimètres interceptent les solutions extragranulaires. Le prélèvement des solutions et le vide partiel des tubes lysimétriques étaient faits après chaque période de pluie à l'aide d'une pompe manuelle. Dans les pédons saturés à l'année longue par la nappe phréatique, des tubes en PVC ont été installés à $50 \mathrm{~cm}$ de profondeur. Ces tubes étaient percés dans les trentes derniers $\mathrm{cm}$ pour s'assurer que les solutions de sol interceptées reflètent les caractéristiques chimiques de celles à la base des profils. La nappe était prélevée à l'aide d'une pompe manuelle, tout en mesurant son niveau avec un fil gradué muni à son extrémité d'un flotteur. Ces tubes ont également été installés à toutes les autres stations lysimétriques du bassin versant pour y observer les variations de la nappe phréatique. Le ruisseau drainant le bassin versant de l'affluent $n^{\circ} 1$ a 
été échantillonné à deux endroits, soit au site 3 à une vingtaine de mètres en amont des transects, et au site 4 à environ 100 mètres en aval (fig. 1).

Les précipitations incidentes, les pluvio-lessivats, les solutions de sol ainsi que l'eau du ruisseau ont été échantillonnés dans des bouteilles en polyéthylène. Les échantillons étaient conservés dans une glacière, jusqu'à ce qu'ils soient entreposés à une température constante de $4{ }^{\circ} \mathrm{C}$, quelques heures suivant leur prélèvement.

Le $\mathrm{pH}$ et la conductivité ont été mesurés dans les 24 heures sur des échantillons non filtrés. Les échantillons d'eau pour les analyses ultérieures étaient passés à travers un filtre en acétate-cellulose de 0,45 $\mu \mathrm{m}$. Environ $100 \mathrm{ml}$ des échantillons filtrés étaient subséquemment transférés dans des bouteilles de $250 \mathrm{ml}$ et acidifiés avec de l'acide nitrique à $0,2 \%$ afin d'éviter la précipitation de $\mathrm{Al}$ et $\mathrm{Fe}$ sur les parois des bouteilles avant l'analyse de l'échantillon. Fe total, Al total et $\mathrm{SiO}_{2}$ ont été analysés par colorimétrie et lecture au spectrophotomètre à lecture directe $\mathrm{HACH}$ (DR/2000). Les concentrations des cations basiques ont été obtenues en utilisant le spectrophotomètre d'absorption atomique de Instrumentation Laboratory ${ }^{\circledR}$ (modèle 157) ; $\mathrm{Na}^{+}$et $\mathrm{K}^{+}$ont été analysés en flamme air-acétylène et $\mathrm{Ca}^{2+}$ et $\mathrm{Mg}^{2+}$ en flamme $\mathrm{N}_{2} \mathrm{O}$-acétylène. Enfin, les anions $\mathrm{SO}_{4}{ }^{2-}, \mathrm{NO}_{3}{ }^{-}, \mathrm{Cl}^{-}$ ont été analysés à l'aide du chromatographe ionique Waters (modèle 510) de Millipore ${ }^{\circledR}$.

\section{4 - RÉSULTATS ET DISCUSSION}

\subsection{Sélection des traceurs}

Dans le bassin versant de l'affluent $n^{\circ} 1$, les concentrations des solutions de sol en $\mathrm{SO}_{4}{ }^{2-}$ et $\mathrm{Cl}^{-}$varient peu avec la profondeur et sont sensiblement les mêmes que celles de la pluie incidente et des pluvio-lessivats, signe que l'eau de pluie est en équilibre chimique avec les sols du bassin versant. En conséquence, ces ions ne sont pas retenus par les sols et migrent jusqu'à la nappe phréatique et au ruisseau. Du simple fait que ces traceurs naturels ne varient pas beaucoup d'un compartiment à l'autre, les graphes de mélange n'ont pas été très informatifs quant à l'identification des compartiments responsables de la qualité de l'eau de l'affluent $n^{\circ} 1$.

Les teneurs en $\mathrm{NO}_{3}{ }^{-}$et $\mathrm{K}^{+}$sont grandement influencées par l'activité biologique. Comme en témoignent les fortes concentrations dans les solutions de sol des horizons organiques et les faibles concentrations du ruisseau, ces ions sont en grande partie absorbés par la végétation du bassin versant. Les processus biologiques ne sont pas directement intégrés au modèle. De ce fait, $\mathrm{NO}_{3}{ }^{-}$et $\mathrm{K}^{+}$ se sont avérés inefficaces afin d'identifier un mélange conservateur quelconque.

Bien que $\mathrm{Al}_{\mathrm{t}}$ ait pu se révéler une espèce chimique intéressante pour identifier un mélange conservateur, surtout dans un bassin versant où la complexolyse est un mécanisme pédogénétique très actif et différenciant nettement les horizons, sa très faible concentration dans le ruisseau indique qu'il précipite en grande partie dans les horizons $\mathrm{B}$ podzoliques sous forme de complexes organo-métalliques et/ou d'hydroxydes amorphes (BÉLANGER et al., 1997). Il est donc difficile d'établir 
une relation entre les concentrations en $\mathrm{Al}_{\mathrm{t}}$ dans les différents compartiments et la concentration en $A l_{t}$ dans le ruisseau. Le fer total est différent parce qu'il est remis en solution par les conditions réductrices des profils de la plaine d'inondation. Ce traceur aurait pu s'avérer utile pour identifier un mélange conservateur, mais comme les mécanismes de précipitation et de remise en solution des composés ferrugineux se succèdent selon l'état hydrique des profils, l'interprétation des résultats devient compliquée et presque impossible à valider. Les concentrations des solutions de sol en $\mathrm{Fe}_{\mathrm{t}}$ sont très variables pour un même compartiment, particulièrement dans les horizons $B$ podzoliques de la plaine d'inondation, où la nappe phréatique les submerge plusieurs fois au cours de l'année, même pendant l'été (BÉLANGER et al., 1997).

Sous les conditions du bassin versant de l'affluent $n^{\circ} 1$, seulement cinq traceurs chimiques naturels sur douze, soit le $\mathrm{pH}, \mathrm{Na}^{+}, \mathrm{Ca}^{2+}, \mathrm{Mg}^{2+}$ et $\mathrm{SiO}_{2}$, démontrent un mélange conservateur. Les graphes de mélange associant la conductivité électrique aux traceurs conservateurs n'ont pas été retenus puisque certains traceurs intervenant eux-même dans la conductivité électrique ont été écartés. Au total, dix graphes de mélange ont donc servi à l'identification des compartiments responsables de la qualité de l'eau du ruisseau.

Compte tenu de la grande variabilité des teneurs de $\mathrm{Ca}^{2+}$ et $\mathrm{Mg}^{2+}$ entre les compartiments, se sont ces ions qui ce sont avérés les plus utiles pour déterminer les sources d'approvisionnement du cours d'eau. II est connu que les eaux qui s'écoulent latéralement le long des versants s'enrichissent progressivement en éléments avant d'atteindre le cours d'eau (LAFOND, 1992). Cet enrichissement graduel des solutions de sol expliquerait la différence marquée des concentrations en $\mathrm{Ca}^{2+}$ et $\mathrm{Mg}^{2+}$ entre les compartiments de haut et de mi-versant et ceux de la plaine d'inondation.

En règle générale, $\mathrm{SiO}_{2}$ n'est pas très mobile dans les sols où règne un pédoclimat froid et humide. Pourtant, elle semble l'avoir été assez pour donner des différences suffisamment marquées entre les compartiments. D'après BÉLANGER et al. (1997), la précipitation de $\mathrm{SiO}_{2}$ avec le fer et l'aluminium sous forme d'hydroxydes amorphes à la base des profils d'une part, et l'altération relativement avancée des horizons d'éluviation d'autre part, expliqueraient la variabilité des concentrations des solutions de sol en $\mathrm{SiO}_{2}$.

Dans les sols du bassin versant, la lente décomposition de la matière organique s'accompagne d'une acidification qui entraîne la production de composés organiques, comme les acides organiques simples, les acides citrique, oxalique, acétique et les polymères phénoliques (ROBERT et al., 1987). Le pH s'élève de haut en bas des profils, suggérant que les composés organiques se chargent (ou se neutralisent) progressivement en éléments métalliques au cours de leur migration dans le sol (BÉLANGER et al., 1997). Mis en relation avec les autres traceurs conservateurs, le $\mathrm{pH}$, qui est donc assez variable à travers les profils du bassin versant, produit une représentation graphique conforme aux autres graphes de mélange.

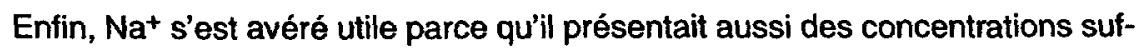
fisamment distinctes d'un compartiment à lautre et ce, même si cet ion n'a pas de comportement particulier dans les sols du bassin versant. 


\subsection{Identification des compartiments}

CAISSIE et al. (1996) ont démontré que l'écoulement de base du ruisseau Catamaran est assuré par la nappe phréatique, autant en périodes d'étiage qu'en périodes de fort débit. Celle-ci constitue donc assurément l'un des trois compartiments approvisionnant le cours d'eau. C'est pourquoi la nappe phréatique a été incluse par défaut dans les graphes de mélange en utilisant la composition chimique la plus représentative du cours d'eau en périodes de faible débit. Pour ce faire, les données chimiques du ruisseau ont été mises en relation avec celles du débit. Au total, trois jours d'échantillonnage (étiage) ont été retenus pour simuler la composition chimique de la nappe phréatique. Á la figure 4, le compartiment " nappe " reflète donc les moyennes des concentrations des traceurs chimiques dans la nappe phréatique durant l'étiage.

La figure 4 montre clairement que ce sont les compartiments de la plaine d'inondation (pédons IV.1 et III.1) qui forment le plus de possibilités de mélange avec la nappe phréatique. Toutefois, la sélection de deux compartiments venant s'ajouter à celui de la nappe phréatique doit se faire de façon systématique. Les possibilités de mélange entre les compartiments de la plaine d'inondation et la nappe phréatique sont nombreuses pour un même graphe de mélange. Pour expliquer la qualité des eaux de surface en utilisant seulement deux compartiments supplémentaires à la nappe phréatique, il faut procéder à une élimination des compartiments dont la contribution au cours d'eau n'est pas nécessairement manifeste pour l'ensemble des traceurs conservateurs. De plus, les compartiments qui montrent une composition chimique semblable à celle du ruisseau ont une plus grande valeur que ceux qui démontrent une composition très différente. La sélection des compartiments doit donc se faire en tenant compte de trois éléments déterminants : les compartiments doivent 1) avoir une composition chimique similaire à celle du ruisseau, 2) circonscrire l'ensemble des variations chimiques du ruisseau dues aux fluctuations du débit, et 3) donner un mélange conservateur pour l'ensemble des traceurs chimiques retenus.

Les compartiments retenus pour la séparation de l'hydrogramme sont les horizons $\mathrm{B}$ de la plaine d'inondation. Bien que l'analyse ne serve pas à valider l'information, EMMA montre le mélange conservateur entre les horizons $B$ des pédons III.1 et IV.1 (versant nord et sud respectivement) et la nappe phréatique (fig. 4). Ici, quelques compartiments possédant une composition chimique semblable à celle du cours d'eau ont été inévitablement rejetés. Par exemple, les horizons $C$ des pédons IV.1 et III.1, qui sont constamment saturés d'eau même pendant la saison estivale, ainsi que les horizons d'éluviation $(A)$ de ces même pédons, s'ajoutent au mélange conservateur, mais dans une moindre mesure que les horizons $B$ des même pédons.

Néanmoins, plusieurs compartiments ont montré une composition chimique très différente de celle du ruisseau. Par exemple, l'opposition entre la composition chimique des solutions de sol des horizons organiques (LFH) et celle du ruisseau, i.e., un faible $\mathrm{pH}$ et l'abondance de $\mathrm{Al}_{\mathrm{t}}$ et de $\mathrm{Fe}_{\mathrm{t}}$ dans les solutions des horizons organiques contre un $\mathrm{pH}$ neutre et de faibles concentrations de $\mathrm{Al}_{\mathrm{t}}$ et de $\mathrm{Fe}_{\mathrm{t}}$ dans le ruisseau, indique que cette voie d'écoulement est rarement empruntée dans le bassin versant de l'affluent $n^{\circ} 1$ (BÉLANGER et al., 1997). De plus, les solutions de sol de haut et de mi-versant sont généralement moins chargés en éléments que le ruisseau puisqu'elles ont été en contact moins longtemps avec les formations superficielles que les solutions de sol à proximité du ruisseau et 

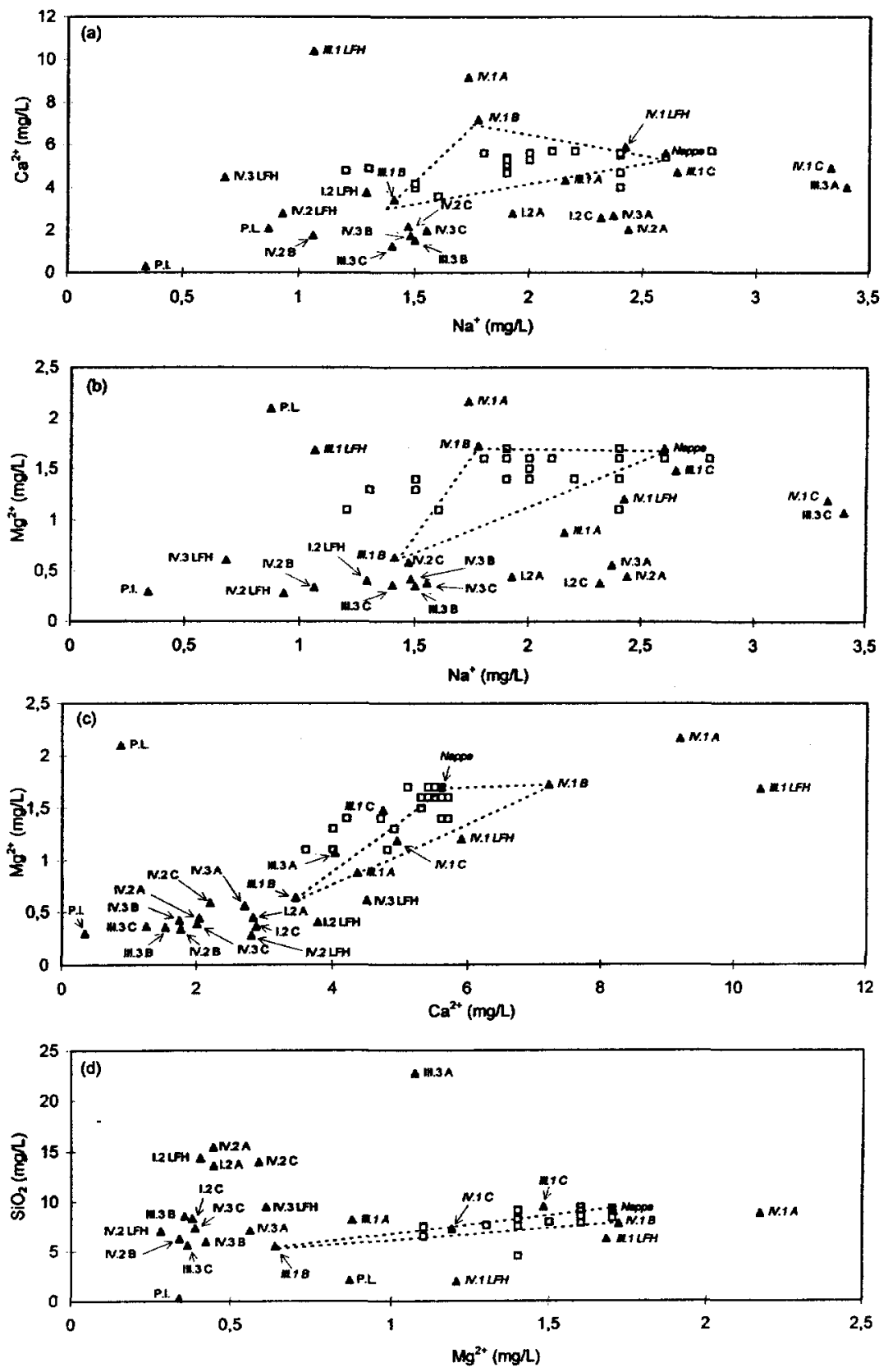

N.B. : Les échantillons du ruisseau sont indiqués par des carrés, la moyenne des compartiments par des triangles. P.I. et P.L. représentent respectivement les moyennes des pluies incidentes et des pluvio-lessivats prélevés dans tous les sites d'étude. N.B. ; Streamwater chemistry is represented by squares, end-members mean values by triangles. P.I. and P.L. represent respectively wet deposition and throughall.

Figure 4 Graphes de mélange de (a) $\mathrm{Ca}^{2+}$ vs $\mathrm{Na}^{+}$, (b) $\mathrm{Mg}^{2+}$ vs $\mathrm{Na}^{+}$, (c) $\mathrm{Mg}^{2+} v s \mathrm{Ca}^{2+}$ et (d) $\mathrm{SiO}_{2} v s \mathrm{Mg}^{2+}$ pour le bassin versant de l'affluent $n^{\circ} 1$ du ruisseau Catamaran.

Mixing diagrams of (a) $\mathrm{Ca}^{2+}$ vs $\mathrm{Na}^{+}$(b) $\mathrm{Mg}^{2+}$ vs $\mathrm{Na}^{+}$, (c) $\mathrm{Mg}^{2+}$ vs $\mathrm{Ca}^{2+}$ and (d) $\mathrm{SiO}_{2} \mathrm{vsMg}^{2+}$ for Catamaran brook tributary $\mathrm{n}^{\circ} 1$ catchment. 
l'eau du ruisseau. De ce fait, l'écoulement hypodermique dans les sols de haut et de mi-versant ne participe pas directement à l'enrichissement du ruisseau, mais alimente plutôt les solutions de sol à proximité du chenal du ruisseau qui l'approvisionnent directement. La signature chimique de la pluie incidente et des pluviolessivats est également très différente de celle de l'affluent $n^{\circ} 1$. Dans ce milieu forestier, et compte tenu de la taille du ruisseau, il semble donc qu'il n'y a aucune précipitation directe dans le cours d'eau sans qu'il n'y ait passage à travers les sols du bassin versant. Tout comme l'écoulement hypodermique dans les sols de haut et de mi-versant, la pluie et le ruissellement de surface ne contribuent pas directement à l'écoulement du ruisseau, mais alimentent plutôt les compartiments à proximité du ruisseau (PEARCE et al., 1986).

Ce sont donc les processus hydrologiques terrestres à proximité du cours d'eau qui contrôlent principalement la qualité de l'eau du ruisseau. En fait, le mélange de l'eau de la nappe phréatique (i.e. l'écoulement souterrain) aux solutions de sol des horizons $B$ de la plaine d'inondation semble expliquer relativement bien la composition chimique du ruisseau, indépendamment des conditions hydrologiques du bassin versant.

La contribution des solutions de sol des horizons B de la plaine d'inondation était inattendue du fait que ceux-ci sont affectés par la présence de la nappe phréatique pendant toute la période sans gel. Conséquemment, les solutions de sol des horizons $B$ auraient pu refléter la composition chimique de la nappe phréatique. Pourtant, la signature chimique de ces solutions de sol diffère clairement de celle de la nappe phréatique, ce qui suggère que la qualité de l'eau de la nappe phréatique à ce niveau des profils est modifiée autant par les réactions chimiques d'altération des sols que par les apports en éléments provenant des horizons supérieurs et transportés par les eaux de percolation.

\subsection{Séparation de l'hydrogramme}

La contribution relative des trois compartiments (i.e., nappe phréatique, IV.1 B et III.1 B) à l'écoulement total du ruisseau a été évaluée à l'aide de l'équation du bilan massique en utilisant simultanément $\mathrm{Mg}^{2+}, \mathrm{Ca}^{2+}, \mathrm{Na}^{+}$et $\mathrm{SiO}_{2}$, lesquels semblent être des traceurs chimiques conservateurs (HOOPER et SHOEMAKER, 1986 ; HOOPER et al., 1990 ; GÉNÉREUX et al., 1993).

Les résultats de la séparation de l'hydrogramme sont présentés sur un histogramme afin d'éviter de faire une trop grande interpolation entre les différents événements étudiés (fig. 5). Ici, les concentrations des traceurs chimiques sont celles mesurées le jour de l'échantillonnage. Les barres se divisent en trois parties, i.e., l'apport de chacun des compartiments à l'écoulement total du ruisseau. La somme de la contribution relative des trois compartiments doit donc égaler le débit (hauteur d'eau) du ruisseau. Une barre représente alors dans son entier le débit total du ruisseau pour une journée d'étude.

L'écoulement souterrain semble assurer l'écoulement de base du ruisseau en période de faible débit (fig. 5). Par exemple, la nappe phréatique contribue à $100 \%$ de l'écoulement du ruisseau pour les jours montrant les plus faibles valeurs de débit, soit le 21 août, le 23 septembre et le 14 octobre. Ce même compartiment assure l'écoulement de base du ruisseau indépendamment des conditions hydrologiques. Toutefois, la contribution relative de la nappe phréatique diminue à mesure que le débit du ruisseau augmente. Dans ce cas, l'écoulement 


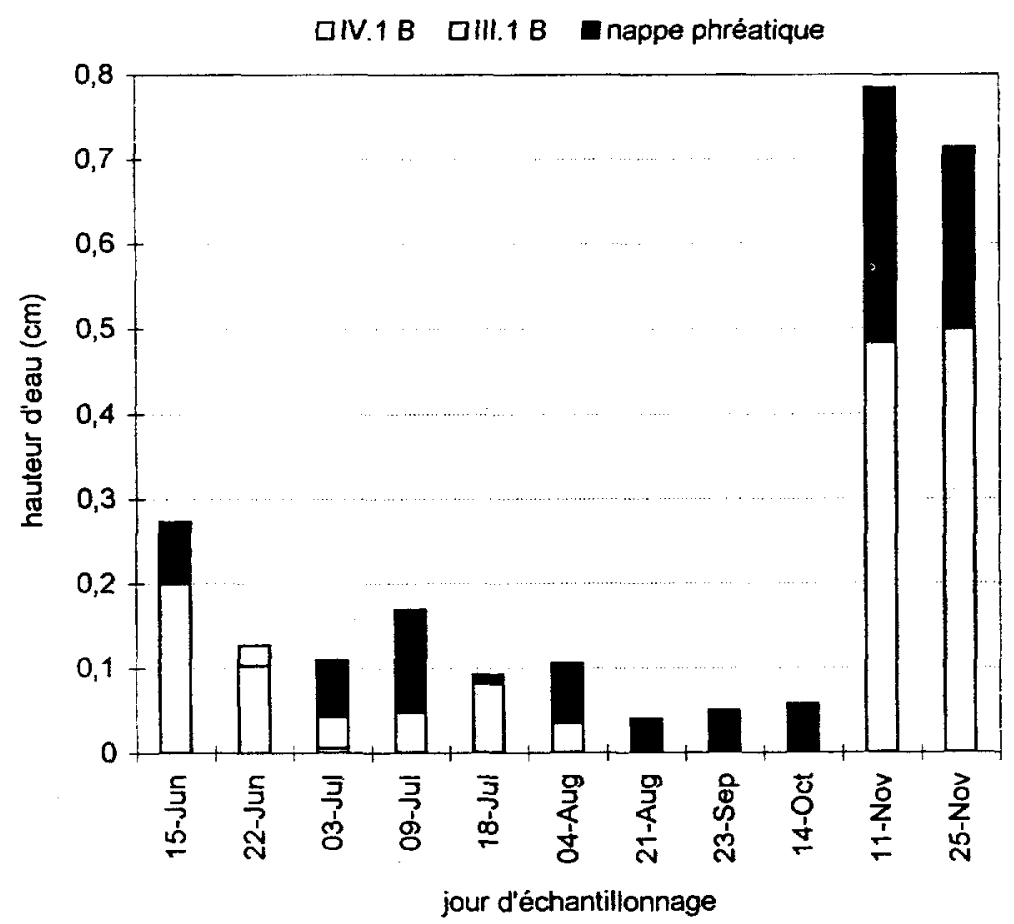

Figure 5 Contribution relative de la nappe phréatique et de l'écoulement dans les horizons $B$ des sols de la plaine d'inondation à l'affluent $n^{\circ} 1$ du ruisseau Catamaran.

Relative contribution of groundwater and subsurface flow from $B$ horizons along the stream banks to Catamaran brook tributary $n^{\circ} 1$.

hypodermique au niveau des horizons $B$ du versant nord (pédon III.1) de la plaine d'inondation contribue pour une large part à l'écoulement total de l'affluent $n^{\circ} 1$. Par exemple, le 25 novembre, les solutions de sol des horizons $B$ du versant nord de la plaine d'inondation ont contribué pour $70 \%$ de l'écoulement total du ruisseau contre $30 \%$ pour la nappe phréatique. Cependant, l'écoulement hypodermique dans les horizons $B$ de l'autre côté du ruisseau n'a alimenté le ruisseau que le 22 juin et le 3 juillet, i.e., lors de faibles débits, avec des proportions de $81 \%$ et $5 \%$, respectivement (fig. 5).

La nappe phréatique affecte les horizons $B$ des deux versants de la plaine d'inondation (fig. 6). Cette observation suggère donc que l'écoulement hypodermique en condition saturée devrait se faire autant dans les horizons B des sols du versant nord que dans ceux du versant sud. Pourtant, la séparation de l'hydrogramme montre que c'est essentiellement l'écoulement hypodermique dans la partie saturée des horizons B de la plaine d'inondation du versant nord qui s'ajoute à la nappe phréatique pour donner l'écoulement total du ruisseau. La figure 6 indique que la nappe phréatique a occupé les horizons $B$ des sols des deux versants du bassin pendant toute la période sans gel, ce qui élimine la possibilité que ce soit les fluctuations du toit de la nappe phréatique qui affectent l'action de ce type d'écoulement. 


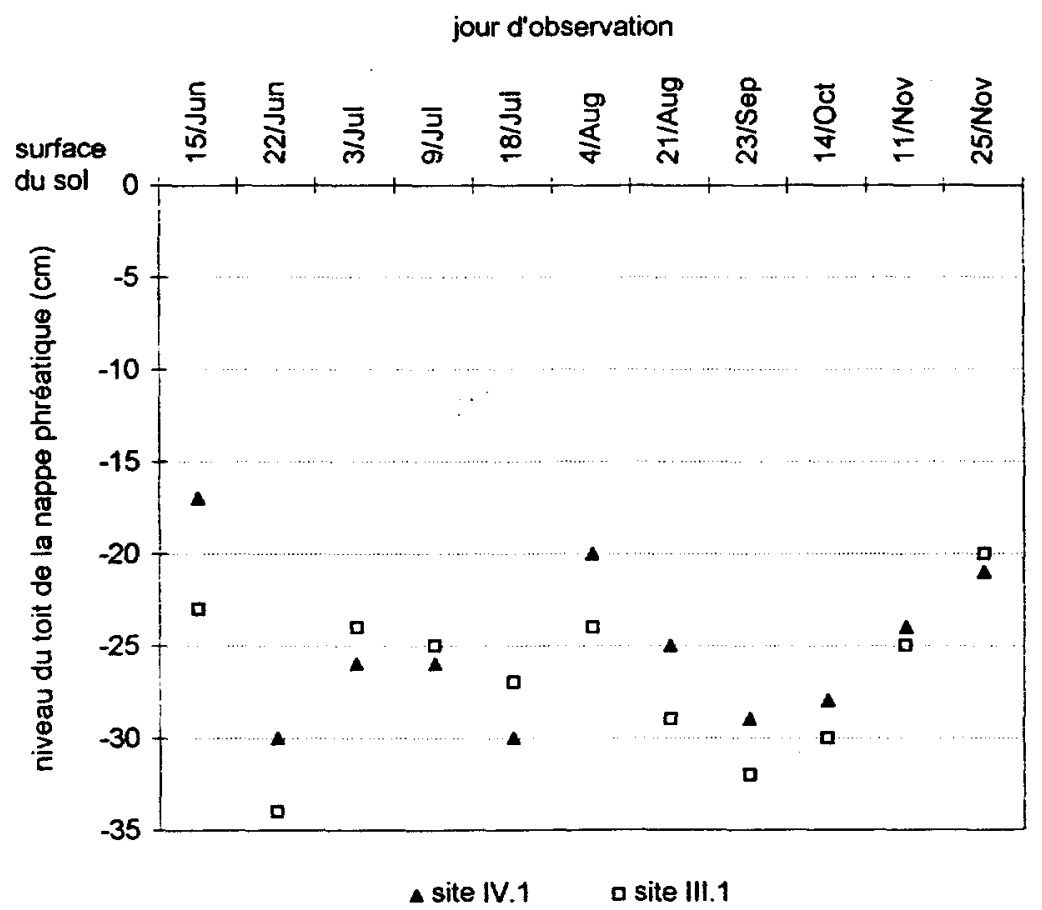

Figure 6 Variation du niveau de la nappe phréatique dans les sols de la plaine d'inondation du bassin versant de laffluent $n^{\circ} 1$ du ruisseau Catamaran (15 juin au 25 novembre 1995).

Groundwater variation in the soils along the stream banks of Catamaran brook tributary $n^{\circ} 1$ (June 15 to November 25, 1995).

Ce que le modèle EMMA permet de dire à ce stade, c'est qu'un compartiment mal défini dans l'espace et ayant une signature chimique semblable à celle de l'horizon $\mathrm{B}$ du pédon du versant nord constitue la source première (avec la nappe phréatique) lors de forts débits. II est donc préférable d'interpréter cette information en terme de type de solution de sol plutôt qu'en terme de provenance physique (versant nord et sud). À cet effet, le cours d'eau est alimenté par les solutions de sol des deux versants du bassin.

\subsection{Analyse du modèle EMMA}

Le modèle EMMA sert à identifier les compartiments responsables de la qualité de l'eau du ruisseau. Toutefois, il ne paraît pas simple de l'utiliser pour calculer la contribution de chacun des compartiments. L'interprétation des résultats de la séparation de l'hydrogramme est laborieuse du fait que seulement trois compartiments sont retenus pour modéliser la qualité de l'eau du cours d'eau. Certains compartiments pouvant s'avérer déterminants dans la qualité de l'eau du ruisseau sont absents. De toute évidence, le ruisseau est alimenté par les solutions de sol provenant des deux versants du bassin. La nappe phréatique affecte la base des profils de la plaine d'inondation pendant toute la saison estivale, ce qui laisse croire que l'écoulement hypodermique au niveau des horizons $\mathrm{B}$ et $\mathrm{C}$ 
alimente le cours d'eau pendant toute la période sans gel. En périodes arrosées, it se peut même que l'écoulement hypodermique dans les horizons superficiels (i.e., LFH et A) de la plaine d'inondation vienne alimenter le ruisseau. II y a donc neuf sources potentielles de la qualité du cours d'eau: la nappe phréatique, les horizons $B$ de la plaine d'inondation (versants nord et sud), les horizons $C$ de la plaine d'inondation (versants nord et sud), les horizons A de la plaine d'inondation (versants nord et sud), et les horizons LFH de la plaine d'inondation (versants nord et sud). Il pourrait y avoir un dixième compartiment avec la contribution de l'affluent $n^{\circ} 1$ en amont des toposéquences de sol (III et IV) et des sites d'échantillonnage du ruisseau (sites 3 et 4).

En somme, la contribution relative des trois compartiments retenus peut être biaisée parce que 1) la sélection des deux transects ne correspond pas nécessairement à des toposéquences représentatives des sols en amont des versants nord et sud, 2) les compartiments retenus n'étaient qu'en partie représentatifs de la totalité des réservoirs alimentant l'affluent $n^{\circ}$ 1, et 3) les pédons IV.1 et III.1 ne sont pas nécessairement représentatifs des sols de la plaine d'inondation. De ce fait, l'application du modèle EMMA dans le bassin versant de l'affluent $n^{\circ} 1$ n'est utile qu'à un niveau qualitatif. II ne donne qu'un aperçu général et simplifié de la contribution des sols à l'écoulement du ruisseau. Un modèle mécaniste développé à partir des variations du niveau de la nappe phréatique et du gradient hydraulique permettrait sans doute de simuler avec plus d'exactitude la qualité des eaux de surface à partir de la composition chimique des solutions de sol.

HENDERSHOT et al. (1992) ont proposé que la qualité des eaux de surface tire normalement son origine des solutions de sol à proximité du chenal du cours d'eau. Ainsi, à mesure que l'eau circule à travers les différents environnements pédochimiques, sa qualité est modifiée. Les résultats du modèle EMMA ont montré que les compartiments présentant une signature chimique très proche de celle de l'affluent $n^{\circ} 1$ sont ceux des pédons de la plaine d'inondation, lesquels représentent la fin du séjour de l'eau dans le bassin versant avant de rejoindre le cours d'eau. Dans le bassin versant de l'affluent $n^{\circ} 1$, le modèle EMMA conserve donc une certaine crédibilité en confirmant ce que HENDERSHOT et al. (1992) ont observé dans le bassin versant de l'Hermine (Québec).

\section{5- CONCLUSION}

L'hypothèse qui sous-tend le développement du modèle EMMA est bonne parce qu'elle distingue un grand nombre de compartiments pouvant potentiellement contribuer à la qualité de l'eau du ruisseau, en autant que ceux-ci soient suffisamment différents les uns des autres. Toutefois, les résultats de l'étude démontrent qu'un mélange plus complexe (i.e., plus que trois compartiments) est nécessaire pour expliquer la qualité de l'eau du ruisseau.

Le modèle EMMA est à un stade de développement qui ne permet pas encore d'évaluer avec précision la contribution relative des différents compartiments. Un modèle mécaniste développé à partir des variations du niveau de la nappe phréatique, du gradient hydraulique et de la composition chimique des solutions de sol permettrait sans doute de reproduire plus rigoureusement l'hydrogramme et les 
chémogrammes du ruisseau. Toutefois, le modèle EMMA est avantageux parce qu'il met clairement en relation la composition chimique des compartiments à celle du ruisseau et enlève parfois tout doute quant à l'action d'un processus susceptible d'alimenter le cours d'eau.

\section{REMERCIEMENTS}

Les auteurs tiennent à remercier les organismes subventionnaires sans qui cette étude n'aurait eu lieu ; la Faculté des Études supérieures et de la Recherche de l'Université de Moncton, le Programme d'aide financière à la recherche et à la création de l'Université du Québec à Montréal, le Programme de Coopération Québec/Nouveau-Brunswick, la papetière Repap et Environnement Canada. Les auteurs tiennent également à remercier les trois réviseurs (anonymes) pour avoir contribué à l'amélioration de l'article.

\section{RÉFÉRENCES BIBLIOGRAPHIQUES}

BAKER, J.P., SCHOFIELD, C.L. (1982). Aluminium toxicity to fish in acidic waters. Water, Air and Soil Pollut., 18, 289-309.

BERGSTRÖM, S., CARLSSON, B., SANDBERG, G., MAXE, L. (1985). Integrated modelling of runoff, alkalinity, and $\mathrm{pH}$ on a daily basis. Nord. Hydrol., 16, 89-104.

BÉLANGER, N., JOLICOEUR, S., BOUCHARD, M. (1997). Variabilité des processus podzoliques en fonction de l'état hydrique des profils et de leur position sur les versants : le cas d'un petit bassin-versant forestier du centre du NouveauBrunswick (Canada). Hommes et Terres du Nord, 3-4, 183-189.

CAISSIE, D., POLLOCK, T.L., CUNJAK, R.A. (1996). Variation in stream water chemistry and hydrograph separation in a small drainage basin. J. Hydrol. 178, 137-157.

CHRISTOPHERSEN, N., SEIP, H.M., WRIGTH, R.F. (1982). A model for streamwater chemistry at Birkenes, Norway. Water Resour. Res., 18, 977-996.

CHRISTOPHERSEN, N., NEAL, C., HOOPER, R.P. (1990). Modelling streamwater chemistry as a mixture of soil water and end-members - a step towards second generation acidification models. $J$. Hydrol., 116, 307-320.

CUNJAK, R.A, CAISSIE, D., EL-JABI, N. (1990). Projet de recherche sur l'habitat du ruisseau Catamaran: description et champ d'étude général. Rapp. tech. Can. sci. halieu. aquat. No. 1751, 15 p.

DINCER, T., PAINE, B.R., FLORKOWSKI, T., MARTINEC, J., TONGIORGI, E. (1970). Snowmelt runoff from measurements of tritium and oxygen-18. Water Resour. Res., 6, 110-124.

GÉNÉREUX, D.P., HEMOND, H.F., MULHOLLAND, P.J. (1993). Use of radon-222 and calcium as tracers in a three-end-member mixing model for streamflow generation on the West Fork of Walker Branch Watershed. J. Hydrol., 142, 167-211.

GHERINI, S.A., MOK, L., HUDSON, R.J.M., DAVIS, G.F., CHEN, C.W., GOLDSTEIN, R.A. (1985). The ILWAS model : formulation and application. Water, Air, Soil Pollut., 26, 425-459.

HALL, R.J., DRISCOLL, C.T., LIKENS, G.E., PRATT, J.M. (1985). Physical, chemical, 
and biological consequences of episodic aluminium addition to a stream. Limnol. Oceanogr., 30, 212-220.

HENDERSHOT, W.H., SAVOIE, S., COURCHESNE, F. (1992). Simulation of streamwater chemistry with soil solution and groundwater flow contributions. J. Hydrol., 136, 237-252.

HOOPER, R.P., SHOEMAKER, C.A. (1986). A comparison of chemical and isotopic hydrograph separation. Water resour. Res., 22, 1444-1454.

HOOPER, R.P., STONE, A., CHRISTOPHERSEN, N., DEGROSBOIS, E., SEIP, H.M. (1988). Assessing the Birkenes model of stream acidification using a multisignal calibration methodology. Water Resour. Res., 24, 1308-1316.

HOOPER, R.P., CHRISTOPHERSEN, N., PETERS, N.E. (1990). Modelling streamwater chemistry as a mixture of soil water end-members - an application to the Panola Mountain catchment, Georgia, U.S.A. J. Hydrol., 116, 321-343.

JOLICOEUR, S., BOUCHARD, M., LAMARCHE, M., FORGET, J. (1996). Couverture pédologique et acquisition de la charge chimique des eaux dans un sous-bassin versant du ruisseau Catamaran. Revue de l'Université de Moncton, 1-2, 185-217.

LAFOND, R. (1992). Larchitecture du sol Dans Pédologie forestière. Éd : Modulo, Mont-Royal (Québec), p.21-35.

LIKENS, G.E., BORMANN, F.H., JOHNSON, N.M., FISHER, D.W., PIERCE, R.S. (1970). The effects of forest cutting and herbicides treatment on nutrient budgets in the Hubbard Brook watershed-ecosystem. Ecol. Monogr., 40, 23-47.

MARTINEC, J. (1975). Subsurface flow from snowmelt traced by tritium. Water Resour. Res., 11, 496-498.

MAULÉ, C.P., STEIN, J. (1990). Hydrologic flow path definition and partitioning of spring meltwater. Water Resour. Res., 26, 2959-2970.

NEAL, C., SMITH, C.J., WALLS, J, DUNN, C.S. (1986). Major, minor, and trace element mobility in the acid upland forested catchment of the upper River Severn, midWales. J. Geol. Soc. London, 143, 635-648.

NODVIN, S.C., DRISCOLL, C.T., LIKENS, G.E. (1988). Soil processes and sulfate loss at the Hubbard Brook Experimental Forest. Biogeochemistry, 5, 185-199.

PEARCE, A.J., STEWART, M.K., SKLASH, M.G. (1986). Storm runoff generation in humid headwater catchments. 1. Where does the water come from? Water Resour. Res., 22, 1263-1272.

PINDER, G.F., JONES, J.F. (1969). Determination of ground-water component of peak discharge from the chemistry of total runoft. Water Resour. Res., 5, 438-445.

PLAMONDON, A.P. (1993). Influence des coupes forestières sur le régime d'écoulement de l'eau et sa qualité ; revue de littérature. Ministère des forêts du Québec, Sainte-Foy, Québec.

RAMPTON, V.N., GAUTHIER, R.C., THIBAULT, J., SEAMAN, A.A. (1984). Memoir 416: Quaternary geology of New Brunswick. Commission géologique du Canada. Ottawa.

ROBERT R., RAZZAGHE M.H., RANGER J. (1987). Rôle du facteur biochimique dans la podzolisation. Études expérimentales sur les mécanismes géochimiques et les évolutions minéralogiques. Dans Podzols et podzolisation, Éds: D. Righi et A. Chauvel, Association Française pour l'Étude du Sol, Plaisir et Paris, p.207-223.

TRANSPORTS CANADA. (1984). Normales climatiques/Canada. Ministère des Transports, Direction de la météorologie, Ottawa.

SCHINDLER, D.W. (1988). Effects of acid rain on freshwater ecosystems : a summary of recent research. Science, 239 : 149-156. 